pagnée d'abondantes chutes de neige rendit tout ravitaillement impossible pendant quelques jours et faillit avoir des suites tragiques.

C'est dans le Rapport pour 1884 que l'Amiral Mouchez prévoit, avec une ferme confiance, d'après les résultats favorables obtenus par MM. Henry, la solution dn problème longtemps cherché de faire la Carte du Ciel à l'aide de la photographie. MM. Henry eurent alors, en effet, recours à cette méthode pour surmonter les difficultés que présentait la construction de la Carte écliptique aux environs de la voie lactée. Dès les premiers essais, avec un objectif de 6 pouces taillé par eux, ils obtinrent de très-remarquables épreuves de plusieurs groupes d'étoiles. On constate en particulier sur la reproduction par l'héliogravure du cliché photographique de l'amas de Persée, obtenu le ro Octobre $\mathbf{1 8 8 4}$, que les images des étoiles jusqu'à la $\mathrm{I}^{\mathrm{mi \theta}}$ et à la $\mathrm{I} 3^{\mathrm{me}}$ grandeur sont venues avec une extrême netteté. Les pointés sur le cliché avaient une précision si grande que plus d'un astronome se montra d'abord sceptique. Des savants de l'étranger vinrent à Paris pour examiner les clichés de leurs propres yeux. Le succès tenait essentiellement, on le devine, à la rare perfection du travail optique de l'objectif. L'amiral Mouchez décida alors la construction immédiate de l'équatorial photographique de $0.34 \mathrm{~m}$ d'ouverture et $3.40 \mathrm{~m}$ de distance focale, qui est devenu le type des instruments choisis par le premier Congrès astrophotographique international réuni trois ans plus tard, en I 887 , à l'Observatoire de Paris.

Dans ce court espace de temps, de 1884 à 1887 , MM. Henry avaient en effet réussi à convaincre les savants - réfractaires parfois à la nouveauté - de la valeur des nouveaux procédés pour l'astronomie physique aussi bien que pour la description du ciel, témoin leur admirable cliché des Pléiades et leurs belles photographies de spectres stellaires. L'appareil de mesure des plaques, construit par M. Gautier, sur leurs indications, montra enfin à tous le haut degré de précision de la méthode photographique. De nombreuses recherches theoriques et expérimentales, accomplies par MiM. Henry, ont contribue à rendre plus efficaces et plus rigoureux les nouveaux procédés d'exploration du ciel.

Ce n'est pas ici le lieu d'entrer dans plus de détails sur la grande entreprise qui, aujourd'hui, est en bonne voie d'exécution tant pour le travail du Catalogue que pour la Carte proprement dite. Mais il faut reconnaitre que l'avance si honorable prise par l'Observatoire de Paris en 1887 a eu pour cause l'ingénieuse et énergique activité de nos deux collègues, et surtout leur entente parfaite des choses de l'optique, qui leur a permis, comme en se jouant, de réaliser des merveilles.

En France, les parties optiques de tous les grands instruments sont sorties de leurs mains, depuis le téléscope de Toulouse de $0.80 \mathrm{~m}$ d'ouverture, pour les débuts, jusqu'aux grandes lunettes de Nice, Paris et Meudon. Enfin, plus de la moitié des objectifs photographiques du monde entier, destinés à la Carte du Ciel, sont leur œuvre.

Des mérites si éclatants avaient assuré à nos deux collègues la haute estime de tous les astronomes. Les distinctions honorifiques arrivèrent par surcroît, tout d'abord de l'étranger. Mais la simplicité qui était le caractère du Collègue que nous regrettons n'en fut pas altérée. Tel je le vois dans mon souvenir, il y a près de 30 ans, lorsque j'entrai en relations avec lui, tel il était resté: modeste, réservé presque à l'excès, s'effaçant volontairement en toute occasion. Sans les Congrès de la Carte du Ciel, on aurait ignoré pour ainsi dire à l'Observatoire la solidité et la variêté des connaissances scientifiques que notre collegue avait acquises par ses efforts personnels, la sûreté et la finesse d'un jugetnent hautement apprécié par les savants qui venaient s'initier à la pratique de la photographie astronomique.

O. Callandreau.

\title{
Berichtigung zu meiner Schrift „Untersuchungen über den Lichtwechsel Algols".
}

Herr Prof. Dr. G. Müller hat mich darauf aufmerksam gemacht, daß die Zahlen der Tafel $V$ im Anhange um nahezu einen Tag fehlerhaft sind. Bei der Fertigstellung der Tafeln für den Druck ist bei der Umarbeitung für $188_{3}$ +0.46 statt -0.46 gesetzt worden; dadurch sind alle Zahlen dieser Tafel um -0.92 Tage zu korrigieren.

Sternwarte Leiden, 1903 Oktober.

Ant. Pannekoek.

(28) Bellona. Korrektion der Ephemeride (Berl. Jahrb. 1905) 1903 Okt. $26:+6^{\mathrm{m}} 37^{\mathrm{s} 82}+25^{\prime}$ 9"2. W. Luther.

(71) Niobe. Korrektion der Ephemeride (Berl. Jahrb. 1905) 1903 Okt. 19: - 165.94-1'33"9. W. Luther.

(148) Gallia. Korrektion der Ephemeride (Berl. Jahrb. 1905) 1903 Okt. $24:+33^{5} \cdot 50+53^{\prime \prime} 4$. F. Pidoux.

Anzeige betreffend Erneuerung des Abonnements siehe Nr. 39 Ir.

Inhalt zu Nr. 3912. E. Goedseels. Vraie signification de l'erreur moyenne. 369. - W. Doberck. On the orbit of $\varphi$ Ursae majoris. $37 \mathrm{r}$. $-W$. Doberck. On the orbit of 99 Herculis = A.C. $15.373 .-C$. Rambaud, $F$. Sy. Observations de planètes. 375. $-A$. Abetti. Pianeta (1 Io) Lydia. 379. - M. Wolf. Photographische Aufnahmen von kleinen Planeten. 379. - S. W. Burnham. Note on Schjellerup 8I44 and BD. $+5^{\circ} 455^{6}$. 379. - Zusatz hierzu. 381. - O. Callandreau. Prosper Henry. 381. - A. Pannekoek. Berichtigung zu meiner Schrift ఎUntersuchungen über den Lichtwechsel Algols $\$$. 383. - Korr. von Planetenephemeriden. $3^{8} 3$. 